\title{
Dynamique de l'occupation du sol dans le Parc National du W et sa périphérie au nord-ouest du Bénin
}

\author{
Julien AVAKOUDJO ${ }^{1 *}$, Adi MAMA ${ }^{1}$, Ismaïla TOKO ${ }^{2}$, Valentin KINDOMIHOU ${ }^{1}$ \\ et Brice SINSIN ${ }^{1}$ \\ ${ }^{1}$ Laboratoire d'Ecologie Appliquée (LEA), Faculté des Sciences Agronomiques (FSA), \\ Université d'Abomey-Calavi (UAC), 01 BP 1651 Abomey-Calavi, Bénin. \\ ${ }^{2}$ Laboratoire de Cartographie (LaCarto), Département de Géographie et Aménagement du Territoire (DGAT), \\ Faculté de Lettres, Arts et Sciences Humaines (FLASH), Université d'Abomey-Calavi (UAC), Benin. \\ *Auteur correspondant, E-mail : julienavakoudjo@yahoo.fr ; Tél :00 22995715586.
}

\section{RESUME}

La dynamique spatio-temporelle de l'occupation du sol et les facteurs de dégradation ont été analysés à partir des images Landsat de 1972, de 1990 et de 2008, de la télédétection et d'un système d'information géographique (SIG), dans le Parc National du W et sa périphérie dans le Nord-Ouest du Bénin. L'objectif de la présente étude est d'une part d'analyser la dynamique de l'occupation du sol entre 1972 et 2008, et d'autre part, de déterminer les facteurs de dégradation du couvert végétal. Des enquêtes socioéconomiques ont été réalisées dans les villages riverains du Parc National du W. La classification supervisée par maximum de vraisemblance a été appliquée. Deux matrices de transition ont permis de mettre en évidence les conversions subies par les différentes unités d'occupation du sol. Les formations forestières ont régressé de 22,70\% à $17,00 \%$ entre 1972 et 2008. Les forêts galeries dégradées quasi inexistantes en 1972 se sont reconstituées à partir de 1990 et sont passées de $0,60 \%$ à 2,85\% en 2008. Quant aux forêts denses ainsi que les forêts claires et savanes boisées, elles ont subi une régression en terme de superficie $(0,23 \%$ en 1972 à $0,09 \%$ en 2008 et de 17,90\% à 12,91\% respectivement). Par contre, les savanes arborées et arbustives ont connu une légère diminution (73\% en 1972 à $66,65 \%$ en 2008) tandis que les mosaïques de champs et jachères ont connu une augmentation de leur superficie (1,38\% en 1972 à 13,97\% en 2008), soit plus de 10 fois leur superficie initiale de 1972. La démographie, l'agriculture et le surpâturage sont les trois principaux facteurs de dégradation du Parc National du $\mathrm{W}$ et de sa périphérie.

(c) 2014 International Formulae Group. All rights reserved.

Mots clés : Télédétection, SIG, dynamique, matrice de transition, Parc National du W.

\section{INTRODUCTION}

La dégradation des terres est liée à la pauvreté et à des pressions humaines, aux régimes fonciers et à la sécheresse qui ont pour corollaires le surpâturage, les activités agricoles non durables, la surexploitation des terres, et la déforestation. Mais la terre fertile se raréfie dans de nombreux pays africains, et la croissance rapide de la population sans un mode de culture agricole intensive conduit à la dégradation des terres (CEA, 2009). L'érosion de la biodiversité constitue de nos jours une menace pour l'humanité (Sinsin et Kampmann, 2010). Dans la plupart de la 
bibliographie scientifique et des discours politiques sur l'érosion de la biodiversité, quatre causes principales s'identifient très souvent: surexploitation, disparition ou transformation des habitats, introductions d'espèces et pollution (Lévêque, 2010), oubliant les causes principales de ce phénomène : démographie, pauvreté, course au profit et incitations économiques de type subvention (Lévêque, 2010). Plus de $70 \%$ des espèces végétales sont menacées de disparition au niveau mondial (Vié et al., 2009). Les effets directs et indirects des activités humaines sur la biodiversité sont des questions sujettes à de nombreux débats scientifiques (Scouvart et Lambin, 2006 ; Toko, 2008). L'Afrique tropicale est la plus durement touchée par cette crise environnementale (FAO, 2001). Le changement net des superficies forestières en Afrique intertropicale est le plus important de toute la planète (FAO, 2001; Toko et al., 2013). La perte annuelle nette de la superficie forestière est estimée à 5,3 millions d'hectares pour toute l'Afrique, soit $0,78 \%$ de la superficie forestière totale (FAO, 2001).

$\mathrm{Au}$ Bénin, les tendances évolutives des formations naturelles montrent une diminution des superficies forestières au profit de celles des savanes et des espaces anthropiques. Ainsi, le couvert végétal est passé de 5761000 ha en 1990 à 4561000 ha en 2010 , soit une perte de $20,8 \%$. Cette perte correspond en moyenne à 60000 ha de couverture forestière par an soit 1,04\% (FAO, 2010 ; Sinsin et Kampmann, 2010). Ainsi, on assiste à la transformation de vastes étendues continues de paysages naturels en paysages humanisés.

L'une des mesures prises pour conserver la diversité biologique et assurer le maintien des fonctions écologiques est la création et la gestion des aires protégées. Ainsi, en 2002, les Parcs Nationaux du W du Bénin et du Burkina Faso ont été ajoutés à celui du Niger pour former la Réserve du Biosphère Transfrontalière du W (RBTW). La création du Parc National du W devrait permettre de conserver la diversité biologique et de protéger le bassin versant de la rivière Alibori. Malheureusement, les pressions anthropiques sur les ressources biologiques du Parc National du W se font de plus en plus intenses et compromettent sérieusement l'équilibre écologique de ce paysage. Depuis une quarantaine d'années, l'augmentation de la densité humaine s'est accélérée avec pour conséquence une intensification de l'exploitation des ressources naturelles (Michelot et Ouédraogo, 2009). La dynamique sociale actuelle dans les zones tampon et de transition de la RBTW montrent de graves conflits entre éleveurs-agriculteursgestionnaires de la RBTW et les transhumants internationaux au Bénin (Michelot et Ouédraogo, 2009). La transhumance, le braconnage et l'exploitation forestière sont pratiqués dans ce Parc National du W (Kagoné, 2004).

Cette étude a donc pour but d'analyser la dynamique de l'occupation du sol du Parc National du W et de sa périphérie entre 1972 et 2008 d'une part, et de déterminer les facteurs de dégradation du couvert végétal d'autre part. Elle veut aussi vérifier l'hypothèse selon laquelle, les formations forestières ont régressé au profit des formations anthropisées que sont les champs et jachères. La dégradation de l'environnement et par conséquent celle des ressources naturelles est aujourd'hui d'autant plus inquiétante qu'elle ne laisse indifférents ni acteurs de développement, ni chercheurs (Akognongbe et al., 2014).

\section{MATERIEL ET METHODES \\ Cadre d'étude}

La zone d'étude est située au nord du Bénin entre $11^{\circ} 26^{\prime}-12^{\circ} 25^{\prime}$ latitude Nord ; $2^{\circ} 48^{\prime}-3^{\circ} 05$ de longitude Est (Figure 1).

Elle couvre une superficie de 6102 $\mathrm{km}^{2}$ et est située dans la zone soudanienne à deux saisons contrastées. La pluviosité moyenne annuelle est de 833,24 $\mathrm{mm}$. Elle est plus proche de la moyenne de la station de Gaya $(843,06 \mathrm{~mm})$ au Niger que celle de la 
station de Kandi $(1035,1 \mathrm{~mm})$ au Bénin (Tableau 1). La température moyenne annuelle est de $28{ }^{\circ} \mathrm{C}$ (ASECNA, 2010). L'harmattan et l'alizé maritime sont les deux types de vents dominants dans la zone d'étude. Le relief est très peu accidenté et constitué majoritairement de bas plateaux (157 m environ) surmontés de reliefs résiduels représentés par les collines de 213 mètres d'altitudes (Mama, 2013). Le substratum géologique est caractérisé par les formations du socle granito-gneissique très ancien et des dépôts du quaternaire (Viennot, 1978). Les sols ferrugineux occupent la grande partie du cadre d'étude. Sur le plan hydrographique, le Parc National du W et sa zone périphérique appartiennent au bassin versant du fleuve Niger. La rivière Alibori $(338 \mathrm{~km})$ est la principale rivière qui alimente la zone. Le Parc National du W appartient au district phytogéographique du Mékrou (Adomou et al., 2006). La végétation est composée de galeries forestières, de forêts claires et savanes boisées, des savanes arborées, arbustives et des mosaïques de champs et de jachères. Le Parc National du W, dans la commune de Karimama, est limité par les Communes de Kandi, Banikoara, Malanville et le Burkina Faso. Ces communes limitrophes sont réputées comme productrices de coton, de maïs et d'igname où le taux d'accroissement censitaire est en moyenne de 4,14\% (INSAE, 2003).

\section{Données cartographiques utilisées et méthodologie \\ Cartes et images satellitaires}

Les données planimétriques utilisées au cours de cette étude sont les cartes topographiques de l'Institut Géographique National au 1/200 000 (Feuilles NC-31-XXIde Kandi ; ND-31-III de Kirtachi ; ND-31-IV de Sabongari; NC-31-XXII de Gaya).Pour les corrections géométriques, trois images satellitaires Landsat prises à différentes dates ont été utilisées : MSS de 1972 (path 192, row 051 et path 192, row 052), TM de 1990 (path 192, row 051 et path 192, row
052), ETM+ de 2008 (path 192, row 051 et path 192, row 052). Ces données ont fourni le matériel brut pour l'élaboration de statistiques sur les changements du couvert végétal et sur la réalisation des trois cartes d'occupation du sol (1972, 1990 et de 2008) du Parc National du W.

Méthodes de traitement des données images Traitements des images satellitaires

Un ré échantillonnage de l'image MSS de 1972 a été réalisé afin de pouvoir la superposer aux autres images TM de 1990 et ETM+ de 2008. Les interprétations ont été réalisées en utilisant des méthodes manuelles conventionnelles pour l'interprétation des séries temporelles d'images satellitaires. Ces images ont été traitées en utilisant une composition classique de trois bandes $(4,3,2)$ en fausses couleurs infrarouges à l'aide du logiciel Erdas Imagine 9.3. Des aires d'entraînement ont été délimitées pour les classifications supervisées par maximum de vraisemblance et pour les validations de ces classifications lors des opérations de véritéterrain.

Exportation vers un Système d'Information Géographique

Après validation des classifications supervisées, chaque image a été intégrée au Système d'Information Géographique (ArcGIS 9.3). Toutes les images ont été archivées dans ArcGIS 9.3 et vectorisées, ce qui a permis aussi de visualiser les interprétations puis de calculer des statistiques au niveau des types d'occupation du sol pour chacune des trois dates. Les cartes d'occupation du sol de 1972, 1990 et de 2008 du Parc National du W ont été aussi réalisées.

\section{Matrice de transition}

Les matrices de transition sont élaborées pour décrire ces changements d'utilisation des terres pendant une période donnée (Schlaepfer, 2002). Elles ont donc permis de mettre en évidence les différentes formes de conversion qu'ont subies les formations végétales entre 1972 et 2008 . Le nombre $\mathrm{X}$ de lignes de la matrice indique le nombre de classes d'occupation du sol au 
temps $t_{0}(1972)$; le nombre $\mathrm{Y}$ de colonnes de la matrice est le nombre de classes d'occupation du sol converties au temps $t_{1}$ (2008) et la diagonale contient les superficies des formations végétales restées inchangées. Les transformations se font donc des lignes vers les colonnes. Les superficies de ces différentes classes d'occupation du sol ont été calculées à partir du croisement des cartes d'occupation du sol de 1972 et de 2008 à l'aide de la fonction Intersect de la boîte à outils Arctoolbox du logiciel ArcGIS 9.3.

Le taux du changement (Tc) de l'occupation du sol entre deux dates a été calculé pour chaque classe d'occupation du sol sur la base de la formule,

$\mathrm{Tc}=\frac{\mathrm{A} 2-\mathrm{A} 1}{\mathrm{~A} 1} 100 \quad$ (Toyi et al., 2013)

où $\mathrm{A} 1$ et $\mathrm{A} 2$ sont respectivement les superficies initiale et finale de la classe d'occupation du sol. Les valeurs positives de Tc indiquent des progressions de la classe d'occupation du sol tandis que les valeurs négatives traduisent les pertes de végétation au niveau de ladite classe.

\section{Données socio-économiques \\ ethnobotaniques \\ Collecte des données}

Des enquêtes socio-économiques et ethnobotaniques sont effectuées à l'aide de questionnaires dans 14 villages de la Commune de Karimama (Pétchinga, Monssey, Loumbou-Loumbou, Kompa, Kompanti, Torioh, Bogo-Bogo, Gorou-Béri, Mamassy-Gourman, Mamassy-Peuhl, Kofounou, Karimama, Birni-Lafia, Kargui) pour analyser le contexte de la dégradation de l'écosystème. Des entretiens structurés et semi-structurés sont conduits auprès des populations riveraines du Parc National du W. Quatre principaux groupes socioculturels sont identifiés dans la commune de Karimama à savoir les Dendi/Djerma, Peulh, Gourmantché et Haoussa. Le choix des villages est fait sur la base de la représentativité des groupes socioculturels dans le milieu. L'échantillon est proportionnel à la taille de chaque groupe socioculturel dans la commune. Tous les quatre groupes socioculturels sont représentés en tenant compte de la représentativité des personnes âgées qui sont supposées capables d'expliquer les changements écologiques intervenus dans le milieu au fil des temps. L'analyse des systèmes agraires et des systèmes de production animale a permis de comprendre les modes de faire valoir la terre et les modes d'élevage au sein des différents groupes socioculturels et leur impact sur la dégradation des forêts et des savanes. Les paysans sont interviewés individuellement. Les investigations ont porté sur: (i) statut de l'enquêté, activités principale et secondaire, tenure foncière; (ii) perception de l'enquêté sur l'érosion et la dégradation des terres, causes et facteurs de la dégradation des terres dans son champ et dans la commune de Karimama; (iii) évaluation des pratiques de gestion des terres et des techniques culturales. $\mathrm{Au}$ total, 136 personnes sont interviewées dont 66 Dendis et Djermas $(48,5 \%), 31$ Gourmanchés $(22,80 \%), 30$ Peulhs $(22,00 \%)$ et 9 Haoussas représentant le dernier groupe $(6,60 \%)$. Les focus groups ont été réalisés pour avoir des informations complémentaires sur les villages à savoir leurs potentialités, les infrastructures, le mode d'utilisation des terres et les techniques culturales.

\section{Traitement des données et analyse statistique}

Dans la présente étude, onze paramètres dont quatre pour les deux premiers niveaux d'évaluation et trois pour le dernier niveau ont été évalués pour mettre en évidence la perception des communautés locales sur la dégradation du Parc National du $\mathrm{W}$ et sa périphérie. Leur répartition se présente comme suit : (i) causes de dégradation des terres: agriculture, exploitation forestière, surpâturage, feux de végétation, (ii) facteurs de la dégradation des terres: pente, ruissellement, type de sol, couverture du sol, (iii) Autres facteurs naturels et humains : climat, démographie, laxisme du forestier. Ensuite le paramètre prioritaire 
responsable de cette dégradation a été identifié par chaque paysan.

Le test d'indépendance Chi-carré de Pearson a été réalisé pour tester si les perceptions des causes de dégradation d'une part et des facteurs la favorisant d'autre part dépendent des groupes socioculturels. Par ailleurs, une analyse factorielle des correspondances (AFC) a été réalisée pour décrire les relations entre les groupes socioculturels et leurs perceptions des causes et facteurs prioritaires.

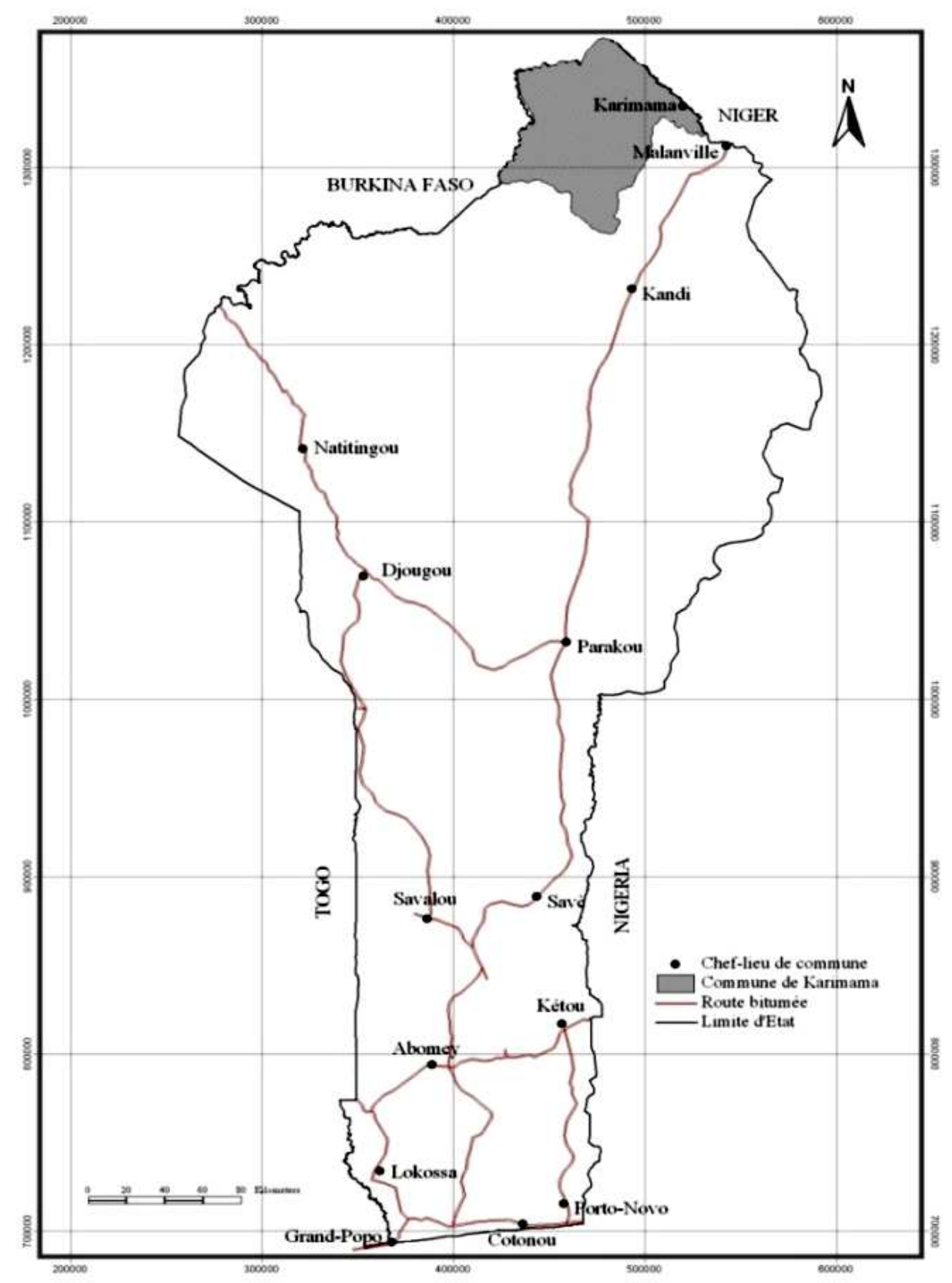

Figure 1 : Localisation de la zone d'étude (Parc National du W) au Bénin. 


\section{RESULTATS}

Composition du paysage et taux de conversion pour les trois périodes 1972$1990 ; 1990-2008$ et 1972-2008

La cartographie de la dynamique des formations végétales et des autres unités d'occupation du sol a été analysée à travers les cartes de végétation de 1972, 1990 et 2008. L'évolution des formations végétales entre ces trois périodes a été ensuite évaluée à travers les taux de changement (Tableau 2). La composition du paysage pour chaque classe d'occupation du sol en 1972, 1990 et 2008 dans la Commune de Karimama et les taux de conversion constatés entre deux dates en $\mathrm{km}^{2}$ et en pourcentage de la superficie totale du paysage sont présentés dans le Tableau 2.

La physionomie de la végétation de la Commune de Karimama était dominée par les savanes arborées et arbustives et parsemée par les forêts claires et savanes boisées en 1972, 1990 et 2008 (Figures 2, 3 et 4). Les savanes arborées et arbustives occupaient environ $73,03 \%, 69,83 \%$ et $66,65 \%$ du territoire de la Commune respectivement en 1972, 1990 et 2008 (Tableau 2) et se répartissent sur l'ensemble de la Commune. Les forêts claires et savanes boisées s'observent un peu partout en des îlots et occupent 17,9\%, 17,74\% et $12,91 \%$ du territoire de la Commune respectivement en 1972,1990 et 2008 (Tableau2). On assiste à l'évolution du front des mosaïques de champs et jachères des agglomérations vers les formations végétales naturelles. De $1,38 \%$ du territoire de la Commune en 1972, ces mosaïques de champs et jachères sont passées à $4,8 \%$ et $13,97 \%$ respectivement en1990 et 2008.

Les taux de changement des formations forestières à savoir (i) forêts galeries, (ii) forêts denses, (iii) forêts claires et savanes boisées et (iv) savanes arborées et arbustives sont négatifs, ce qui traduit une perte de végétation au niveau de ces classes d'occupation du sol durant les trois périodes d'analyse (1972-1990, 1990-2008 et 1972-
2008), tandis que des valeurs positives sont enregistrées pour le même taux de changement en ce qui concerne les plantations et les mosaïques de champs et jachères, ce qui indique des évolutions de ces deux classes d'occupation du sol au cours des mêmes périodes.

\section{Dynamique de l'occupation du sol du Parc National du W et sa périphérie}

La conversion des formations végétales et autres unités d'occupation du sol de 1972 à 2008 est synthétisée dans le Tableau 3. Il ressort de ce tableau que sur les $276,22 \mathrm{~km}^{2}$ de forêts galeries en 1972, 239,15 km² $(86,6 \%)$ sont demeurées inchangées; le reste s'étant transformé en forêts galeries dégradées $(37,07$ $\mathrm{km}^{2}$ ) en 1990.

Sur la période allant de 1990 à 2008, $72,11 \mathrm{~km}^{2}$ de forêts galeries sont demeurées tels quels; le reste s'étant converti en forêts galeries dégradées $\left(156,92 \mathrm{~km}^{2}\right)$ et en mosaïques de champs et jachères $\left(10,22 \mathrm{~km}^{2}\right)$. Des tendances similaires sont obtenues pour la période globale 1972-2008. Les forêts galeries dégradées issues de la perturbation des forêts galeries en 1990, sont demeurées inchangées à $46,05 \%$ de 1990 à 2008 , et le reste $(53,95 \%)$ est transformé en mosaïques de champs et jachères. Au niveau des forêts denses d'une superficie de $13,89 \mathrm{~km}^{2}$, la stabilité est obtenue sur 58,89\% de 1972 à 1990. La dégradation forestière est orientée vers les forêts claires et savanes boisées $(28,15 \%)$, les savanes arborées et arbustives $(12,17 \%)$ et les mosaïques de champs et de jachères $(0,79 \%)$.

De 1990 à 2008, des forêts denses ont connu une stabilité de permanence de $24,0 \%$ dans le paysage, la dynamique régressive de la végétation s'est manifestée par une dégradation des forêts claires et savanes boisées $(28,69 \%)$, des savanes arborées et arbustives $(42,46 \%)$ et des champs et jachères $(4,84 \%)$. Les mêmes tendances sont observées pour la période globale de 1972-2008 au niveau des forêts denses. Les forêts claires et savanes boisées quant à elles, ont été plus 
stables $(98,07 \%)$ avec quelques conversions en savanes arborées et arbustives $(1,56 \%)$ et en forêts denses dans une moindre mesure $(0,37 \%)$. Sur les $1082,36 \mathrm{~km}^{2}$ de forêts claires et savanes boisées en 1990, 201,70 km² (18,63\%) sont restés inchangés en 2008. Une régénération des forêts denses a été observée $(0,24 \%)$; les autres transformations sont orientées vers les savanes arborées et arbustives (73,05\%), les mosaïques de champs et jachères $(8,00 \%)$ et les agglomérations $(0,07 \%)$ pour cette même période.

Pour la période globale 1972-2008, $18,44 \%$ des forêts claires et savanes boisées sont restées stables suivi d'une densification des forêts denses de $0,42 \%$. Une stabilité élevée a également été observée au niveau des savanes arborées et arbustives (94,98\%). Les mosaïques de champs et jachères en évolution croissante ont gardé, quelle que soit la période d'analyse considérée, une dynamique évolutive.

\section{Perception locale sur les causes et facteurs de dégradation du Parc National du W et de sa périphérie}

En fonction des groupes socioculturels de la Commune de Karimama on observe que pour $19,70 \%$ du groupe majoritaire Dendi/Djerma, c'est la démographie qui est la cause prioritaire tandis que pour $29,03 \%$ de Gourmantché, $32,26 \%$ de Fulani et $22,00 \%$ de Haoussa, ce sont respectivement le surpâturage, l'agriculture et le type de sol avec le laxisme des forestiers qui sont indexés comme paramètres induisant la dégradation du Parc National du $\mathrm{W}$ et de sa périphérie (Tableau 4). Cependant, les résultats du test d'indépendance Chi-carré de Pearson ont révélé que les perceptions des causes de dégradation $($ Chi-Sq $=10,56, \mathrm{DF}=12, \mathrm{P}$ Value $=0,567)$ et des facteurs favorisants la dégradation des terres $(\mathrm{Chi}-\mathrm{Sq}=10,03, \mathrm{DF}=$ $24, \mathrm{P}-$ Value $=0,994$ ) ne dépendaient pas des groupes socioculturels étudiés.
Toutefois, en ce qui concerne les causes prioritaires suivant les groupes socioculturels, les résultats de l'analyse factorielle des correspondances (Figure 5, 95\% des informations prises en compte par les trois premiers axes) révèlent que ces causes prioritaires pour les Haoussas sont le laxisme du forestier et le type de sol par opposition aux Peulhs qui pensent plutôt que c'est l'agriculture la cause prioritaire (Axe factoriel 1, Figure 5). Selon les Dendi/Djerma, les causes prioritaires sont la démographie, l'exploitation forestière (coupe du bois), le couvert végétal et les feux de végétation contrairement aux Gourmantchés qui pensent que c'est plutôt le surpâturage et le climat les causes prioritaires (Axe factoriel 2, Figure 5). L'axe factoriel 3 de la même figure oppose les Peulhs pour qui la cause prioritaire est l'agriculture aux Gourmantché qui ont rapporté que les causes prioritaires sont en plus de l'élevage et le climat (axe factoriel 1), le couvert végétal et les feux de végétation.

En effet, la rareté et/ou le retard des pluies, les vents violents sont aussi des facteurs explicatifs des changements climatiques dégradant davantage le Parc National du W et sa périphérie. Selon ces populations, la pente, le ruissellement et les feux de végétation ne causent pas de dommages irréversibles aux écosystèmes du Parc National du $\mathrm{W}$ et de sa zone périphérique. Pour elles, le laxisme du forestier et la non application des textes en vigueur de façon stricte ont contribué à l'accroissement de ces principales activités à l'intérieur du Parc National du W au Bénin. Les agriculteurs, en exploitant la zone tampon, étendent leurs activités agricoles à l'intérieur du Parc National du W. Les éleveurs transhumants y trouvent également refuge. Enfin, la sécheresse et la croissance démographique ont un grand impact négatif sur la dégradation du Parc National du W et de sa périphérie, de l'avis des communautés riveraines. 


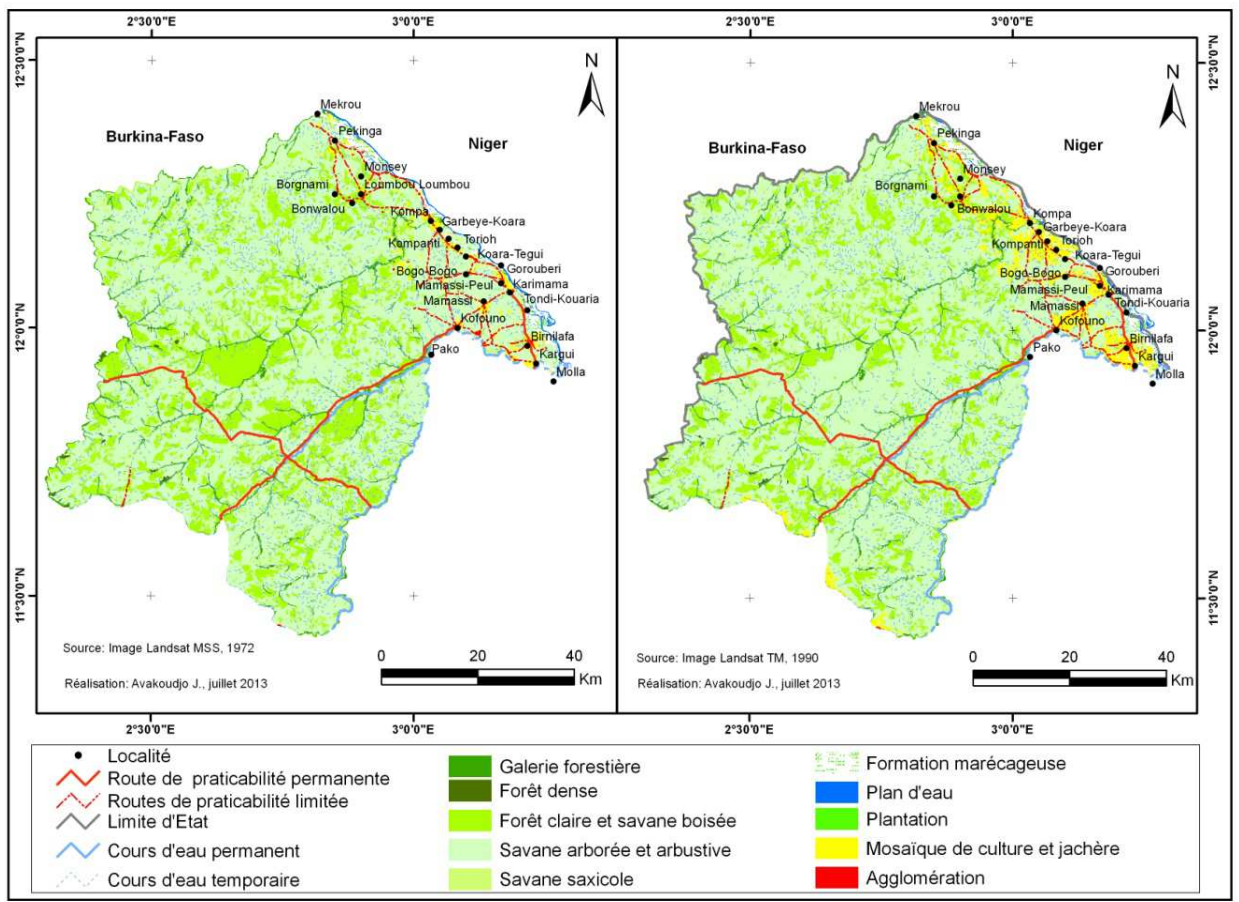

Figure 2 : Dynamique de l'occupation du sol dans la Commune de Karimama de 1972 à 1990.

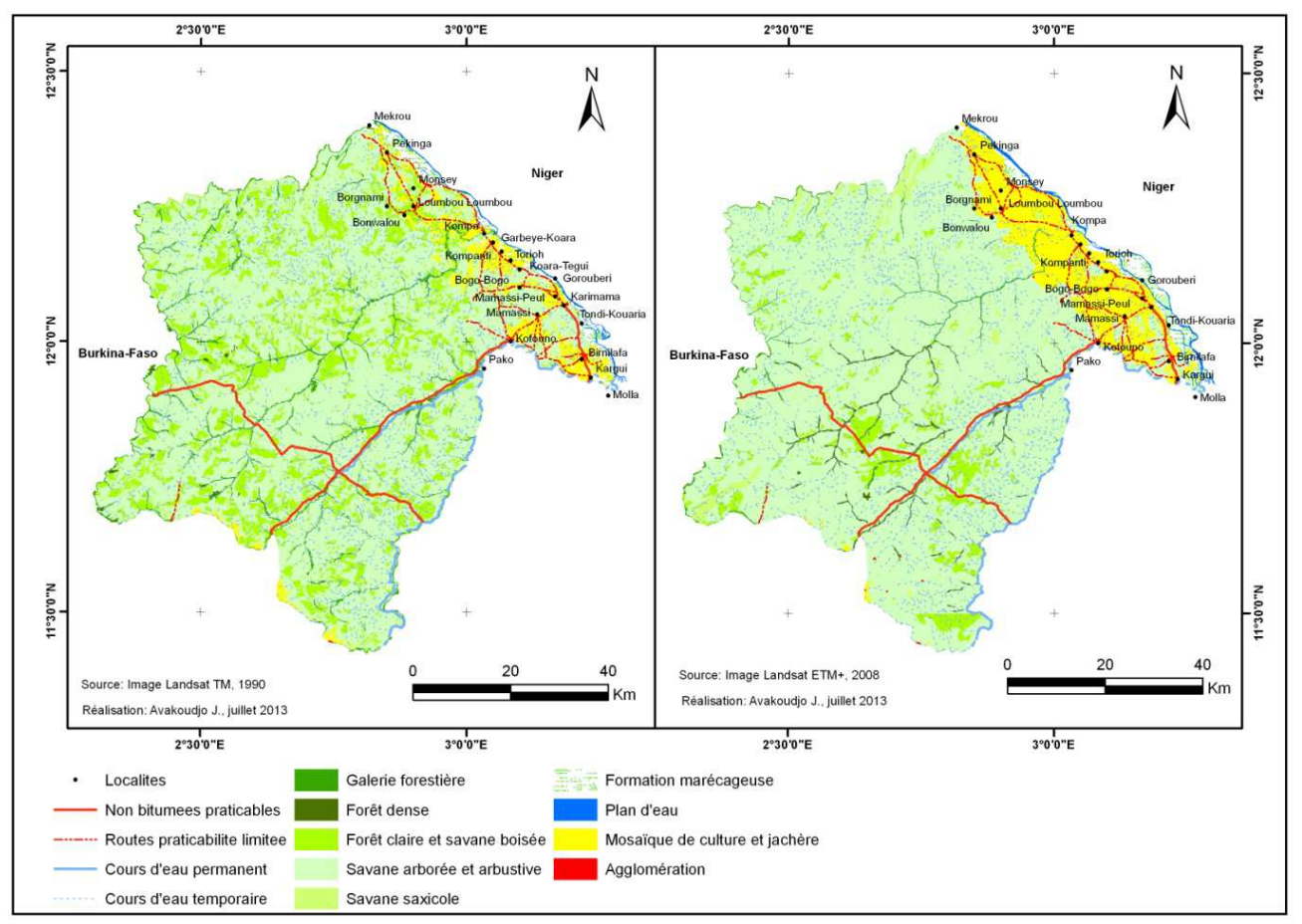

Figure 3 : Dynamique de l'occupation du sol dans la Commune de Karimama de 1990 à 2008. 


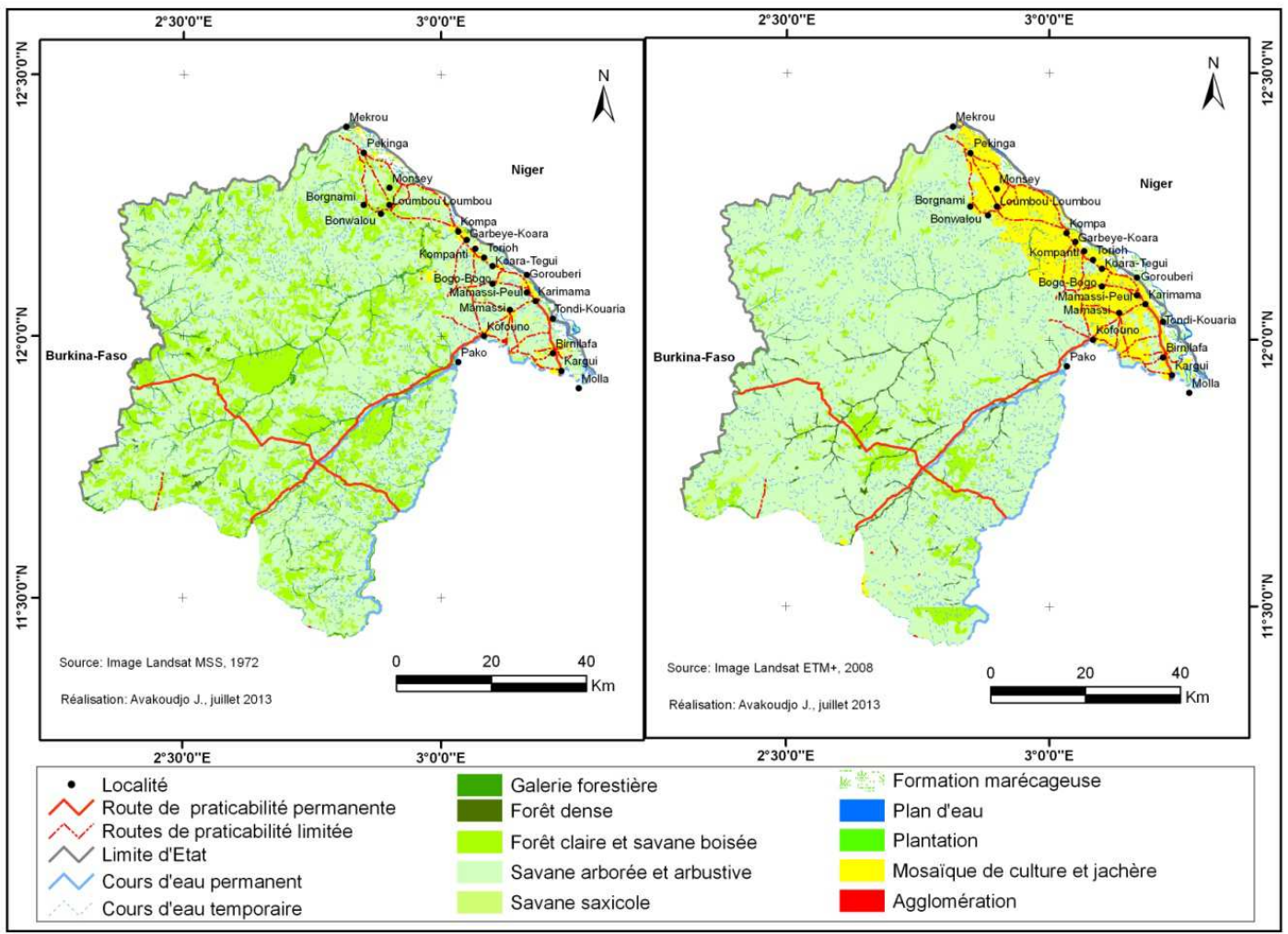

Figure 4 : Dynamique de l'occupation du sol dans la Commune de Karimama de 1990 à 2008.
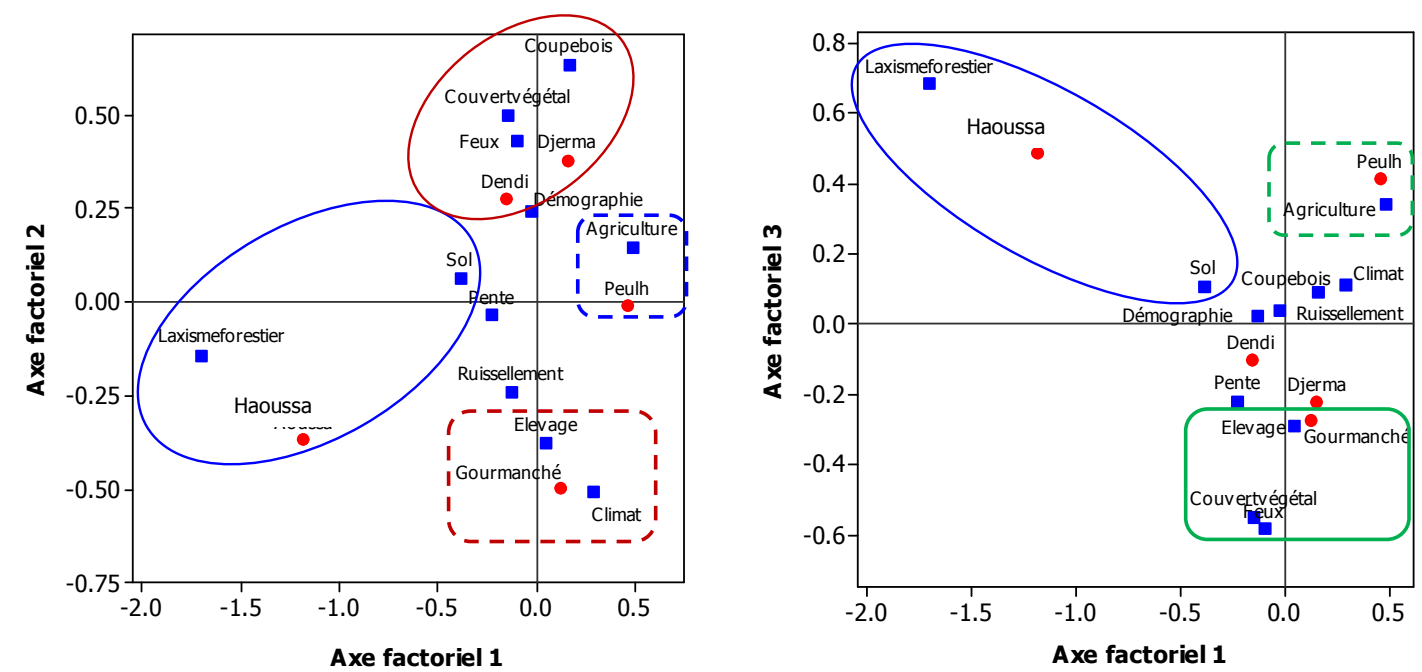

Laxismeforestier : Laxisme du forestier ; Coupebois : Exploitation forestière ; Elevage : Surpâturage Couvertforestier : Couvert forestier ; sol : Type du sol ; feux : Feux de végétation.

Figure 5 : Perception des communautés locales sur les causes et facteurs de dégradation du Parc National du W et de sa périphérie. 
J. AVAKOUDJO et al. / Int. J. Biol. Chem. Sci. 8(6): 2608-2625, 2014

Tableau 1 : Données météorologiques de la zone d'étude.

\begin{tabular}{lccccc}
\hline Données météo & Station & $\mathbf{2 0 0 8}$ & $\mathbf{2 0 0 9}$ & $\mathbf{2 0 1 0}$ & Moyenne \\
\hline \multirow{2}{*}{ Précipitation $(\mathrm{mm})$} & Kandi & 1173,30 & 889,1 & 1042,90 & 1035,10 \\
& Karimama & - & $\mathbf{6 9 1 , 2 3}$ & $\mathbf{9 7 5 , 2 4}$ & $\mathbf{8 3 3 , 2 4}$ \\
& Gaya & 804,2 & 838,3 & 886,7 & 843,06 \\
\hline Gaya & Mini & 22,7 & 23,8 & 23,7 & 23,4 \\
\hline \multirow{2}{*}{ Température $\left({ }^{\circ} \mathrm{C}\right)$} & Max & 35,3 & 36,2 & 36,4 & 35,97 \\
\hline \multirow{2}{*}{ Hygrométrie \% } & Mini & 32 & 33 & 25 & 30 \\
mm : millimètre $;{ }^{\circ} \mathrm{C}:$ degré Celsius ; \% : pourcentage & 64 & 66 & 58 & 62,67 \\
Source : Données de terrain 2009-2010; ASECNA Bénin Niger 2006-2010. & &
\end{tabular}

Tableau 2 : Composition du paysage pour chaque classe d'occupation du sol en 1972, 1990 et 2008 (Commune de Karimama) et les taux de changement pour les trois périodes (1972-1990, 1990-2008 et 1972-2008) en Km² et en \% de la superficie totale du paysage.

\begin{tabular}{|c|c|c|c|c|c|c|c|c|c|}
\hline \multirow{3}{*}{ Classes d'occupation du sol } & \multicolumn{6}{|c|}{ Années } & \multicolumn{3}{|c|}{ Changement \% } \\
\hline & \multicolumn{2}{|c|}{1972} & \multicolumn{2}{|c|}{1990} & \multicolumn{2}{|c|}{2008} & \multirow[t]{2}{*}{ 1972-1990 } & \multirow{2}{*}{$1990-2008$} & \multirow{2}{*}{$1972-2008$} \\
\hline & $\mathbf{k m}^{2}$ & $\%$ & $\mathbf{k m}^{2}$ & $\%$ & $\mathbf{k m}^{2}$ & $\%$ & & & \\
\hline FG & 276,22 & 4,53 & 239,25 & 3,92 & 72,11 & 1,18 & $-13,38$ & $-69,86$ & $-73,89$ \\
\hline FGD & 0,00 & 0,00 & 37,07 & 0,61 & 173,99 & 2,85 & - & 369,35 & - \\
\hline FD & 13,89 & 0,23 & 12,20 & 0,20 & 5,52 & 0,09 & $-12,17$ & $-54,75$ & $-60,26$ \\
\hline FCSB & 1091,98 & 17,90 & 1082,36 & 17,74 & 787,78 & 12,91 & $-0,88$ & $-27,22$ & $-27,86$ \\
\hline SAA & 4456,48 & 73,03 & 4261,08 & 69,83 & 4067,02 & 66,65 & $-4,38$ & $-4,55$ & $-8,74$ \\
\hline SS & 45,61 & 0,75 & 45,61 & 0,75 & 45,15 & 0,74 & 0,00 & $-1,01$ & $-1,01$ \\
\hline FM & 97,13 & 1,59 & 97,13 & 1,59 & 62,84 & 1,03 & 0,00 & $-35,30$ & $-35,3$ \\
\hline Plan d'eau & 25,72 & 0,42 & 25,72 & 0,42 & 21,53 & 0,35 & 0,00 & $-16,29$ & $-16,29$ \\
\hline Plant & 0,71 & 0,01 & 0,71 & 0,01 & 1,05 & 0,02 & 0,00 & 47,89 & 47,89 \\
\hline MCJ & 84,50 & 1,38 & 292,71 & 4,80 & 852,25 & 13,97 & 246,4 & 191,16 & 908,58 \\
\hline $\mathrm{AG}$ & 9,75 & 0,16 & 8,15 & 0,13 & 12,75 & 0,21 & $-16,41$ & 56,44 & 30,77 \\
\hline Total & 6102 & 100 & 6102 & 100 & 6102 & 100 & & & \\
\hline
\end{tabular}


J. AVAKOUDJO et al. / Int. J. Biol. Chem. Sci. 8(6): 2608-2625, 2014

Tableau 3 : Matrice de transition des unités d'occupation du sol de 1972 à 2008 en km².

\begin{tabular}{|c|c|c|c|c|c|c|c|c|c|c|c|c|}
\hline Unités d'occupation du sol & FG & FGD & FD & FCSB & SAA & SS & FM & Plan d'eau & Plant & MCJ & AGGLO & Superficie \\
\hline \multicolumn{13}{|l|}{$1972-1990$} \\
\hline FG & 239,15 & 37,07 & 0,00 & 0,00 & 0,00 & 0,00 & 0,00 & 0,00 & 0,00 & 0,00 & 0,00 & 276,22 \\
\hline FGD & 0,00 & 0,00 & 0,00 & 0,00 & 0,00 & 0,00 & 0,00 & 0,00 & 0,00 & 0,00 & 0,00 & 0,00 \\
\hline FD & 0,00 & 0,00 & 8,18 & 3,91 & 1,69 & 0,00 & 0,00 & 0,00 & 0,00 & 0,11 & 0,00 & 13,89 \\
\hline FCSB & 0,00 & 0,00 & 4,02 & 1070,96 & 17,00 & 0,00 & 0,00 & 0,00 & 0,00 & 0,00 & 0,00 & 1091,98 \\
\hline SAA & 0,00 & 0,00 & 0,00 & 7,49 & 4232,93 & 0,00 & 0,00 & 0,00 & 0,00 & 216,06 & 0,00 & 4456,48 \\
\hline SS & 0,00 & 0,00 & 0,00 & 0,00 & 0,00 & 45,61 & 0,00 & 0,00 & 0,00 & 0,00 & 0,00 & 45,61 \\
\hline FM & 0,00 & 0,00 & 0,00 & 0,00 & 0,00 & 0,00 & 97,13 & 0,00 & 0,00 & 0,00 & 0,00 & 97,13 \\
\hline Plan d'eau & 0,00 & 0,00 & 0,00 & 0,00 & 0,00 & 0,00 & 0,00 & 25,72 & 0,00 & 0,00 & 0,00 & 25,72 \\
\hline Plant & 0,00 & 0,00 & 0,00 & 0,00 & 0,00 & 0,00 & 0,00 & 0,00 & 0,71 & 0,00 & 0,00 & 0,71 \\
\hline MCJ & 0,00 & 0,00 & 0,00 & 0,00 & 9,46 & 0,00 & 0,00 & 0,00 & 0,00 & 75,04 & 0,00 & 84,50 \\
\hline AGGLO & 0,10 & 0,00 & 0,00 & 0,00 & 0,00 & 0,00 & 0,00 & 0,00 & 0,00 & 1,50 & 8,15 & 9,75 \\
\hline \multicolumn{13}{|l|}{ 1990-2008 } \\
\hline FG & 72,11 & 156,92 & 0,00 & 0,00 & 0,00 & 0,00 & 0,00 & 0,00 & 0,00 & 10,22 & 0,00 & 239,25 \\
\hline FGD & 0,00 & 17,07 & 0,00 & 0,00 & 0,00 & 0,00 & 0,00 & 0,00 & 0,00 & 20,00 & 0,00 & 37,07 \\
\hline FD & 0,00 & 0,00 & 2,93 & 3,50 & 5,18 & 0,00 & 0,00 & 0,00 & 0,00 & 0,59 & 0,00 & 12,20 \\
\hline FCSB & 0,00 & 0,00 & 2,60 & 201,70 & 790,68 & 0,00 & 0,00 & 0,00 & 0,00 & 86,66 & 0,72 & 1082,36 \\
\hline SAA & 0,00 & 0,00 & 0,00 & 582,57 & 3271,15 & 0,00 & 0,00 & 0,00 & 0,00 & 405,34 & 2,02 & 4261,08 \\
\hline SS & 0,00 & 0,00 & 0,00 & 0,00 & 0,00 & 45,15 & 0,00 & 0,00 & 0,00 & 0,45 & 0,01 & 45,61 \\
\hline FM & 0,00 & 0,00 & 0,00 & 0,00 & 0,00 & 0,00 & 58,31 & 3,34 & 0,00 & 35,47 & 0,00 & 97,13 \\
\hline Plan d'eau & 0,00 & 0,00 & 0,00 & 0,00 & 0,00 & 0,00 & 4,53 & 18,19 & 0,00 & 3,01 & 0,00 & 25,72 \\
\hline Plant & 0,00 & 0,00 & 0,00 & 0,00 & 0,00 & 0,00 & 0,00 & 0,00 & 0,24 & 0,47 & 0,00 & 0,71 \\
\hline MCJ & 0,00 & 0,00 & 0,00 & 0,00 & 0,00 & 0,00 & 0,00 & 0,00 & 0,81 & 290,05 & 1,85 & 292,71 \\
\hline AGGLO & 0,00 & 0,00 & 0,00 & 0,00 & 0,00 & 0,00 & 0,00 & 0,00 & 0,00 & 0,00 & 8,15 & 8,15 \\
\hline 1972-2008 & & & & & & & & & & & & \\
\hline
\end{tabular}


J. AVAKOUDJO et al. /Int. J. Biol. Chem. Sci. 8(6): 2608-2625, 2014

\begin{tabular}{|c|c|c|c|c|c|c|c|c|c|c|c|c|}
\hline FG & 72,11 & 173,99 & 0,00 & 0,00 & 0,00 & 0,00 & 0,00 & 0,00 & 0,00 & 29,99 & 0,13 & 276,22 \\
\hline FGD & 0,00 & 0,00 & 0,00 & 0,00 & 0,00 & 0,00 & 0,00 & 0,00 & 0,00 & 0,00 & 0,00 & 0,00 \\
\hline FD & 0,00 & 0,00 & 0,91 & 3,76 & 8,18 & 0,00 & 0,00 & 0,00 & 0,00 & 1,04 & 0,00 & 13,89 \\
\hline FCSB & 0,00 & 0,00 & 4,61 & 201,39 & 824,71 & 0,00 & 0,00 & 0,00 & 0,00 & 60,55 & 0,72 & 1091,99 \\
\hline SAA & 0,00 & 0,00 & 0,00 & 582,63 & 3217,68 & 0,00 & 0,00 & 0,00 & 0,00 & 651,23 & 4,94 & 4456,48 \\
\hline SS & 0,00 & 0,00 & 0,00 & 0,00 & 0,00 & 45,15 & 0,00 & 0,00 & 0,00 & 0,44 & 0,01 & 45,61 \\
\hline FM & 0,00 & 0,00 & 0,00 & 0,00 & 0,00 & 0,00 & 58,31 & 4,37 & 0,00 & 34,44 & 0,00 & 97,13 \\
\hline Plan d'eau & 0,00 & 0,00 & 0,00 & 0,00 & 0,00 & 0,00 & 4,53 & 17,16 & 0,00 & 4,04 & 0,00 & 25,72 \\
\hline Plant & 0,00 & 0,00 & 0,00 & 0,00 & 0,00 & 0,00 & 0,00 & 0,00 & 0,51 & 0,14 & 0,06 & 0,71 \\
\hline MCJ & 0,00 & 0,00 & 0,00 & 0,00 & 16,44 & 0,00 & 0,00 & 0,00 & 0,54 & 64,06 & 3,46 & 84,50 \\
\hline $\mathrm{AG}$ & 0,00 & 0,00 & 0,00 & 0,00 & 0,00 & 0,00 & 0,00 & 0,00 & 0,00 & 6,33 & 3,42 & 9,75 \\
\hline
\end{tabular}

FG : Forêts galeries ;FGD : Forêts galeries dégradées ; FD : Forêts denses ; FCSB : Forêts claires et savanes boisées ; SAA : Savanes arborées et arbustives ; SS : Savanes saxicoles :

FM :Forêts marécageuses ; Plant : Plantations ;MCJ : Mosaïque de champs et jachères ;AG : Agglomérations.

Tableau 4 : Pourcentage de réponses positives des différents groupes socioculturels de Karimama concernant les paramètres de dégradation du Parc National du W et de sa zone périphérique.

\begin{tabular}{|c|c|c|c|c|c|c|c|}
\hline Paramètres de dégradation du Parc National du W et sa zone périphérique & Dendi/Djerma & Gourmantché & Fulani & Haoussa & Total & $\%$ & Scores \\
\hline Agriculture & 9 & 3 & 10 & 0 & 22 & 16,18 & 0,16 \\
\hline Climat & 3 & 6 & 5 & 1 & 15 & 11,03 & 0,11 \\
\hline Exploitation forestière & 5 & 0 & 2 & 0 & 7 & 5,15 & 0,05 \\
\hline Couvert végétal & 5 & 1 & 0 & 0 & 6 & 4,41 & 0,04 \\
\hline Démographie & 13 & 3 & 5 & 1 & 22 & 16,18 & 0,16 \\
\hline Surpâturage & 8 & 9 & 3 & 1 & 21 & 15,44 & 0,15 \\
\hline Feux de végétation & 4 & 1 & 0 & 0 & 5 & 3,68 & 0,04 \\
\hline Laxisme du forestier & 2 & 0 & 0 & 2 & 4 & 2,94 & 0,03 \\
\hline Pente & 6 & 3 & 1 & 1 & 11 & 8,09 & 0,08 \\
\hline Ruissellement & 4 & 3 & 2 & 1 & 10 & 7,35 & 0,07 \\
\hline Sol & 7 & 2 & 2 & 2 & 13 & 9,55 & 0,10 \\
\hline Total & 66 & 31 & 30 & 9 & 136 & 100 & 1 \\
\hline
\end{tabular}

$\%$ : pourcentage 


\section{DISCUSSION}

\section{Dynamique de l'occupation du sol}

L'évaluation de la dynamique spatiotemporelle de l'occupation du sol dans le Parc National du W et de ses terroirs riverains montre une amplitude régressive de $37,38 \%$ en 36 ans des formations forestières (forêts galeries, forêts denses et forêts claires savanes boisées). La superficie des champs et jachères est multipliée par dix entre 1972 et 2008 . Les formations forestières font place de plus en plus aux formations anthropisées (champs et jachères). Ces résultats sont conformes à ceux de Mama et al. (2013) dans la zone soudanienne au nord du Bénin, ainsi qu'à ceux de Arouna (2002), Bogaert et al. (2011), Diallo et al. (2011), Orékan (2007) qui montrent une régression continue des forêtssavanes au profit des champs-jachères, sols nus et agglomérations à partir d'étude diachronique. La création de nouveaux champs se fait au détriment des écosystèmes forestiers et savanicoles (Sounon-Bouko et al., 2007). Aussi, l'accroissement des terres agricoles dans le but de nourrir une population sans cesse croissante justifie la réduction de la durée des jachères, l'augmentation d'année en année des espaces anthropisés (Avakoudjo et al., 2011). En effet, dans la zone d'étude, le taux d'accroissement de la population est estimé à 3,58\% par an (INSAE, 2003). La croissance démographique peut entraîner la dégradation des terres dans le court terme, mais il peut aussi stimuler l'innovation et l'intensification agricole ainsi que l'adoption de techniques de conservation (Boserup, 2002). La surexploitation des terres est considérée comme la principale cause de la dégradation du couvert végétal et de réduction de la biodiversité (Sounon-Bouko et al., 2007). Ce phénomène s'observe dans plusieurs régions d'Afrique de l'Ouest (Darkoh, 2003; Ariori et Ozer, 2005 ; Sawadogo et al., 2008). La Commune de Karimama est occupée par le Parc National du $\mathrm{W}$ sur environ $92 \%$ de sa superficie, soit $5632,8 \mathrm{~km}^{2}$ sur $6102 \mathrm{~km}^{2}$ (SNV, 2004), ce qui oblige les paysans à orienter le front agricole vers l'intérieur du Parc National du W au Bénin. La pression agricole voire démographique est telle que la période de la jachère est inexistante, la reconstitution des éléments nutritifs perdus lors des récoltes ne se fait pas, ce qui accélère la chute des paramètres physico-chimiques du sol face à cette surexploitation des potentialités des terres. On assiste aussi à la perte importante des terres arables. Ces terres dégradées produisent moins de nourriture, réduisent la disponibilité de biomasse combustible, rendent les écosystèmes plus résistants, et augmentent la malnutrition et la susceptibilité à la maladie au sein des populations locales. A l'instar de ce qui se passe dans de nombreux pays africains, la terre étant rare, la croissance rapide de la population sans culture intensive, conduit à la dégradation des terres (Hountondji, 2008). Près de $40 \%$ des Africains vivent sur des terres fragiles, la plus grande part dans le monde (Hountondji, 2008). Là où la terre est abondante, la croissance rapide de la population ne conduit pas à la dégradation des terres parce que les agriculteurs changent leurs modes de culture, laissant les terres cultivées en jachère pour reconstituer les éléments nutritifs perdus. Mais la terre est rare dans de nombreux pays africains, et la croissance rapide de la population sans culture intensive conduit à la dégradation des terres.

Une partie des savanes arborées et arbustives a évolué en forêts claires et savanes boisées et une autre partie a régressé en mosaïque de champs et jachères (MCJ). Cette dynamique évolutive est aussi constatée avec cette dernière unité d'occupation du sol MCJ dont une partie a évolué en savanes arborées et arbustives au cours de ces 36 années. Plusieurs auteurs ont souligné que la flore et la végétation d'une région est le résultat d'un long processus de sélection naturelle sous l'action du climat, des conditions édaphiques et topographiques, sans oublier les activités anthropiques (Adedire, 2000; Orthmann, 
2005; Parmentier et al., 2005; Toko, 2008 ; Toko, 2013 ; Sandjong et al., 2013). Sous l'effet des facteurs naturels et anthropiques, les formations végétales changent de physionomie dans le temps. La physionomie des formations végétales est influencée par deux facteurs : les changements climatiques et les activités humaines (Diallo et al., 2011). Les premiers sont considérés comme inéluctables à l'échelle du siècle (Fabien et al., 2006), et leurs effets sur la disparition d'espèces sont généralement limités (Darkoh, 2003). En revanche, l'impact des activités humaines est susceptible d'être à l'origine de modifications de la succession végétale et de la dégradation du milieu (Dembélé, 2012). Dans le Parc National du W, le relief, les sols et les précipitations sont les éléments naturels qui peuvent influer de façon significative sur l'évolution des formations végétales. Les sols de la région de Karimama peuvent être rangés en deux grands groupes: (i) les sols ferrugineux tropicaux caractéristiques de la zone et (ii) les sols hydromorphes. Les premiers sont largement dominants. Selon l'évolution de la roche mère, on y rencontre les sols du continental terminal à concrétion sur grès impropres aux activités agricoles qui s'étendent de Kompa à Monsey (Gandonou et al., 2006). Les sols sans concrétions sur matériaux issus de sédiment sablo-argileux (plus répandus) qui s'étendent de Kompa à Birni-Lafia et les sols sur matériaux kaoliniques rencontrés dans les localités s'étendant entre Tondi-Bouka et Koffonou sont compatibles avec la biologie de nombreuses cultures annuelles (maïs, mil, sorgho, igname, riz pluvial, niébé, etc.) (Gandonou et al., 2006). De façon générale, ces sols ferrugineux tropicaux ont une fertilité qui dépend largement de l'horizon superficiel. Lorsque cet horizon est pauvre en matière organique, alors la fertilité de ces sols est compromise.

\section{Perception locale des causes de la dégradation du Parc National du W et de sa périphérie}

La démographie, l'agriculture et le surpâturage constituent les principales causes de la dégradation du Parc National du W et de sa zone périphérique selon les communautés locales de Karimama. En effet, la taille des actifs agricoles des ménages affecte de manière significative la décision des ménages à défricher de nouvelles terres pour l'agriculture (Houessou et al., 2013). Ainsi, la croissance interne de la population des ménages implique l'accroissement du nombre de personnes à nourrir, de sorte qu'il faut plus de revenus et davantage de produits agricoles. En réponse à cette exigence, les agriculteurs décident souvent de défricher de nouveaux champs afin de surmonter la charge de plus en plus croissante de leurs ménages. Ceci est en accord avec les résultats de Orékan (2007) et Ouédraogo et al., (2010) qui ont conclu au terme de leurs travaux qu'il existe une forte corrélation entre la croissance démographique et la dégradation des terres. Les principales causes de l'érosion et de la dégradation des terres selon plus de $75 \%$ des enquêtés dans la commune de Karimama sont l'agriculture et la déforestation (Avakoudjo et al., 2011). Dans le sud du Parc National du W au Bénin, Houessou et al., (2013) ont trouvé que l'agriculture est la principale force motrice du changement de la couverture végétale dans la région. Notre résultat est donc cohérent avec les études antérieures (Lambin et al., 2003; Wood et al., 2004) qui ont conclu que l'agriculture demeure le principal facteur induisant des changements de la couverture végétale en Afrique subsaharienne. Cependant, les perceptions peuvent varier d'une région à l'autre à l'intérieur du pays. Arouna et al., (2011) ont constaté que la production de charbon de bois représente l'activité principale induisant le changement de la couverture dans le centre du Bénin tandis que Lykke (2000) a signalé les feux intensifs fréquents et la diminution des 
précipitations en tant que facteurs induisant le changement de végétation dans la zone semiaride du Sine Saloum au Sénégal.

La mise en œuvre des activités dans la zone tampon du Parc National du W s'avère nécessaire pour l'application des principes de cogestion et l'exercice des droits d'usage. Considérant les besoins en ressources bois et produits forestiers non ligneux (PFNL) exprimés par la population locale, il peut être développé, dans cette zone, des activités alternatives agro-sylvo-pastorales génératrices de revenus. Le renforcement des mesures de protection doit cependant se faire et l'organisation de patrouilles ou contrôles nocturnes, surtout en saison sèche est vivement recommandée. En cas d'infractions, des sanctions prévues par la réglementation en vigueur doivent être appliquées étant donné que les communautés locales considèrent le laxisme du forestier comme un élément de dégradation du Parc National du $\mathrm{W}$ et de sa périphérie.

\section{Conclusion}

L'étude de la dynamique de l'occupation du sol du Parc National du W et de sa périphérie au Bénin a permis de mettre en évidence les différentes formes de conversion subies par la végétation à partir de la matrice de transition. Ainsi, on peut noter la régression des formations forestières avec une amplitude de 37,38\% pendant 36 ans de 1972 à 2008. Les formations forestières ont cédé progressivement place en partie aux formations anthropisées. La démographie, l'agriculture et le surpâturage constituent les principales causes de dégradation du Parc National du W et de sa périphérie au Nord Ouest du Bénin. Il est donc important d'améliorer le système de production agricole et le mode d'élevage à mettre en œuvre autour de cette aire protégée en adéquation avec l'accroissement de la population pour un développement harmonieux et durable.

\section{REMERCIEMENTS}

Ce travail a été réalisé grâce à l'appui financier et logistique du Laboratoire d'Ecologie Appliquée de la Faculté des Sciences Agronomiques, Université d'Abomey-Calavi (Bénin), de l'Union Européenne (FP6 INCO-dev 031685) à travers le Projet SUN (Sustainable Use of Natural Vegetation in West Africa) et du Gouvernement Béninois à travers la bourse de formation des formateurs. Les Auteurs remercient le Docteur Toyi Mireille et le Professeur Jan Bogaert pour leur contribution.

\section{REFERENCES}

Adedire MO. 2000. Environmental implications of tropical deforestation. $J$. Sustain. Dev. World Ecol., 9: 33-40.

AdomouAC, Sinsin B, Van Der Maesen LJG. 2006 Phytosociological and chorological approaches to phytogeography: a mesoscale study in Benin. Syst. Geogr. Pl., 76: 155-178.

Akognongbe A, Abdoulaye D, Vissin EW, Boko M. 2014. Dynamique de l'occupation du sol dans le bassin versant de l'Ouémé à l'exutoire de Bétérou (Bénin). Afrique Science, 10(2): 228-242.

Ariori SL, Ozer P. 2005. Evolution des ressources forestières en Afrique de l'Ouest soudano-sahélienne au cours des 50 dernières années. Geo. Eco. Trop., 29: 61-68.

Arouna O, Toko I, Djogbénou CP, Sinsin B. 2011. Comparative analysis of local populations' perceptions of socioconomic determinants of vegetation degradation in sudano-guinean area in Benin (West Africa). Int. J. Biodivers. Conserv., 3: 327-337.

Arouna O. 2002. L'exploitation des ressources biologiques et la dynamique de la forêt classée de l'Alibori supérieur au Bénin. Mémoire de Maîtrise de géographie, FLASH, Université d'Abomey-Calavi, Abomey-Calavi, p. 115. 
ASECNA. 2010. Agence pour la Sécurité de la Navigation Aérienne en Afrique et à Madagascar. Station météorologique de Kandi, Bénin.

Avakoudjo J, Kindomihou V, Sinsin B. 2011. Farmers' perception and response to soil erosion while abiotic factors are the driving forces in Sudanian Zone of Benin.Agric. Engineering Res. J., 1(2): 20-30.

Bogaert J, Barima YSS, Ji J, Jiang H, Bamba I, Iyongo WML, Mama A, NyssenE, Dahdouh-Guebas F, Koedam N. 2011.A methodological framework to quantify anthropogenic effects on landscape pattern. In Landscape Ecology in Asian Cultures, Hong S-K, Wu J, Kim J-E, Nakagoshi N (eds). Springer, Verlag: New York; 141-167.

Boserup E. 2002. Technological society and its relation to global environmental change. In Encyclopedia of Global Environmental Change, Social and Economic Dimensions of Global Environmental Change (vol 5), Munn T, Timmermann P (eds). John Wiley and Sons Ltd, 28: New York; 86-96.

CEA. 2009. Développer l'agriculture en Afrique par des chaînes de valeur régionales. Rapport économique sur l'Afrique. Commission Economique des Nations Unies pour l'Afrique, p.179.

Darkoh MBK, 2003. Regional perspectives on agriculture and biodiversity in drylands of Africa. J. Arid Environ., 54 : 261-79.

Dembélé K. 2012. Alternative possible à la production traditionnelle du coton en Afrique dans une perspective de développement durable? Le cas du système de production biologique et équitable au Mali. Thèse de Doctorat, Université de Liège Gembloux Agro. Bio. Tech., Gembloux, p.198.

Diallo H, Bamba I, Barima YSS, Visser M, Ballo A, Mama A, Vranken I, Maiga M, Bogaert J. 2011.Effets combinés du climat et des pressions anthropiques sur la dynamique évolutive de la dégradation d'une aire protégée du Mali (la Réserve de Fina, Boucle du Baoulé). Sécheresse, 22: 97-107.

Fabien A, Waziri MM, Dimitri DB, Frank G. 2006. Dégradation des ressources végétales au contact des activités humaines et perspectives de conservation dans le massif de l'Aïr (Sahara, Niger). Rev. Sci. Env., 7: 1-12.

FAO. 2001. Evaluation des ressources forestières mondiales 2000. Rapport principal, Etude FAO, forêts, FAO: Rome, 140p.

FAO. 2010. Global Forest Resources Assessment 2010: Final Report. FAO Forestry paper 163, FAO, Rome.

Gandonou M, Bossou S, Zannou O, Hounkpèvi D. 2006. Schéma Directeur d'Aménagement de la Commune de Karimama. Rapport de Consultation PRODECOM/Union Européenne. CARTOGEST, $90 \mathrm{p}$.

Houessou LG, Téka O, Toko II, Lykke AM, Sinsin B. 2013. Land use and land cover change at "W" Biosphere Reserve and its surroundings areas in Benin Republic (West Africa). Env. Nat. Res. Resear., 3(2): 87-101.

Hountondji HYC. 2008. Dynamique environnementale en zones sahélienne et soudanienne de l'Afrique de l'Ouest: Analyse des modifications et évaluation de la dégradation du couvert végétal. Thèse de Doctorat, Université de Liège, Belgique, p.131.

INSAE. 2003. Troisième Recensement Général de la Population et de l'Habitation (RGPH-3) au Bénin: Synthèse des Analyses. Direction des Etudes Démographiques, Institut National de la Statistique et de l'Analyse Economique: Cotonou.

Kagoné H. 2004. Etat des lieux de la transhumance dans la zone d'influence du Parc W du fleuve Niger, Rapport de 
synthèse, Programme PARC-W / ECOPAS, 1-24.

Lambin EF, Geist HJ, Lepers E. 2003. Dynamics of land-use and land-cover change in tropical regions. Annu. Rev. Env. Res., 28: 205-241.

Lévêque C. 2010. Biodiversité : réflexion et controverses sur une nature en danger. Les causes de l'érosion de la biodiversité;1-15. http://www. futurasciences.com/ magazines/ environnement/ infos/dossiers/ d/developpement- durable-biodiversite-

Lykke AM. 2000. Local perceptions of vegetation change and priorities for conservation of woody savanna vegetation in Senegal. J. Environ. Manag., 59: 107-120.

Mama A, Sinsin B, De Cannière C, Bogaert J. 2013. Anthropisation et dynamique des paysages en zone soudanienne au nord du Bénin. Tropicultura, 31(1): 78-88.

Mama A. 2013. Anthropisation des paysages au Bénin : dynamique, fragmentation et développement agricole. Thèse de Doctorat, Université Libre de Bruxelles, Belgique, p.198.

Michelot A, Ouédraogo B. 2009. Aires Protégées transfrontalières : le cadre juridique de la réserve de biosphère transfrontalière du W (Bénin, Burkina Faso, Niger).http://cmsdata.iucn.org/ downloads/w_biosphere_fr.pdf

Orékan VA. 2007. Implémentation du modèle local CLUE-s aux transformations spatiales dans le Centre Bénin aux moyens de données socio-économiques et de télédétection. Thèse de doctorat, Université de Bonn, Bonn, Suisse, p.204.

Orthmann B. 2005. Vegetation ecology of a woodland savanna mosaic in central Benin (West Africa): Ecosystem analysis with a focus on the impact of selective logging. PhD thesis, University of Rostock, Rostock, p. 148.

Ouédraogo I, Tigabu M, Savadogo P, Compaore H, Oden PC, Ouadba JM.
2010. Land cover change and its relation with population dynamics in Burkina faso, West Africa. Land Degrad. Dev., 21: $453-462$.

Parmentier I, Stevart T, Hardy OJ. 2005. The inselberg flora of Atlantic Central Africa. I. Determinants of species assemblages. $J$. Biogeo., 32: 685 - 696.

Sandjong Sani RC, Ntoupka M, Ibrahima A, Vroumsia T. 2013. Essai d'analyses de la conception paysanne de l'évolution, de la gestion et de l'utilité du Parc National de Mozogo-Gokoro (Cameroun) en vue de son aménagement. Int. J. Biol. Chem. Sci., 7(6): 2490-2503.

Sawadogo H, Zombre PN, Bock L, Lacroix D. 2008. Evolution de l'occupation du sol de Ziga dans le Yatenga (Burkina Faso) à partir de photos aériennes. Revue de Télédétection, 8: 59-73.

Schlaepfer R. 2002. Analyse de la dynamique du paysage. Fiche d'enseignement 4.2. Lausanne. Laboratoire de gestion des écosystèmes (GECOS), École polytechnique fédérale de Lausanne, Lausanne, p.11.

Scouvart M, Lambin EF. 2006. Approche systémique des causes de la déforestation en Amazonie brésilienne : syndromes, synergies et rétroactions. Environnement, 3: 241-254.

Sinsin B, Kampmann D. 2010. Atlas de la Biodiversité de l'Afrique de l'Ouest. Tome I: Bénin. Cotonou et Frankfurt/Main.

SNV. 2004. Plan de Développement Communal de la Commune de Karimama. SNV : Karimama ; 88.

Sounon Bouko B, Sinsin B, Goura Soulé B. 2007. Effets de la dynamique d'occupation du sol sur la structure et la diversité floristique des forêts claires et savanes au Bénin. Tropicultura, 25(4): 221-227.

Toko I. 2008. Etude de la variabilité spatiale de la biomasse herbacée, de la phénologie et de la structure de la végétation le long 
des toposéquences du bassin supérieur du fleuve Ouémé au Bénin. Thèse de Doctorat, Université d'Abomey-Calavi au Bénin, Abomey-Calavi, p. 241.

Toko IM, Toko II, Gbègbo MC, Sinsin B. 2013. Structure et composition floristiques des forêts denses sèches de la région des Monts Kouffè au Bénin. J. Appl. Biosci., 64 : 4787-4796.

Toko Imorou I. 2013. Effets des facteurs abiotiques sur la répartition spatiale des groupements végétaux dans la zone de transition soudano-guinéenne du Bénin. Int. J. Biol. Chem. Sci., 7(6): 2178-2192.

Toyi MS, Barima YSS, Mama A, André M, Bastin J-F, De Cannière C, Sinsin B, Bogaert J. 2013.Tree Plantation Will Not
Compensate Natural Woody Vegetation Cover Loss in the Atlantic Department of Southern Benin. Tropicultura, 31: 62-70.

Vié JC, Hilton-Taylor C, Stuar NS. 2009. Wildlife in a Changing World: An Analysis of the 2008 IUCN Red List Threatened Species. IUCN: Gland, Switzerland.

Viennot M. 1978. Notice Explicative de la Carte Pédologique de Reconnaissance de la République Populaire du Bénin à 1/200.000, Feuille de Bémbéréké. ORSTOM: Paris, France.

Wood EC, Tappan GG, Hadj A. 2004. Understanding the drivers of agricultural land use change in south-central Senegal. J. Arid Environ., 59: 565-582. 